\title{
Observation of Long Spin Relaxation Times in Bilayer Graphene at Room Temperature
}

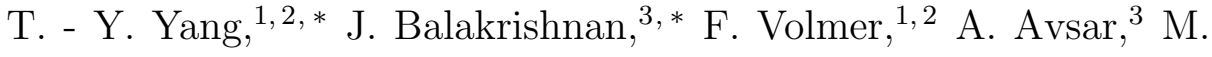 \\ Jaiswal, ${ }^{3}$ J. Samm, ${ }^{1,2}$ S. R. Ali, ${ }^{1,2}$ A. Pachoud,${ }^{3}$ M. Zeng, ${ }^{3}$ M.

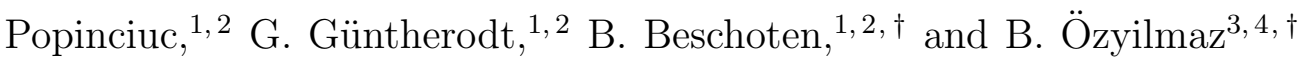 \\ ${ }^{1}$ II. Institute of Physics, RWTH Aachen University, 52074 Aachen, Germany \\ ${ }^{2} J A R A$ : Fundamentals of Future Information Technology, 52074 Aachen, Germany \\ ${ }^{3}$ Department of Physics, National University of Singapore, Singapore 117542, Singapore \\ ${ }^{4}$ NanoCore, National University of Singapore, Singapore 117576, Singapore
}

(Dated: October 30, 2018)

\begin{abstract}
We report on the first systematic study of spin transport in bilayer graphene (BLG) as a function of mobility, minimum conductivity, charge density and temperature. The spin relaxation time $\tau_{s}$ scales inversely with the mobility $\mu$ of BLG samples both at room temperature (RT) and at low temperature (LT). This indicates the importance of D'yakonov - Perel' spin scattering in BLG. Spin relaxation times of up to 2 ns at RT are observed in samples with the lowest mobility. These times are an order of magnitude longer than any values previously reported for single layer graphene (SLG). We discuss the role of intrinsic and extrinsic factors that could lead to the dominance of D'yakonov-Perel' spin scattering in BLG. In comparison to SLG, significant changes in the carrier density dependence of $\tau_{s}$ are observed as a function of temperature.
\end{abstract}

PACS numbers: 85.75.-d, 72.25.Dc, 72.25.Rb, 72.80.Vp

*These authors contributed equally to this work

†Electronic address: barbaros@nus.edu.sg, bernd.beschoten@physik.rwth-aachen.de 

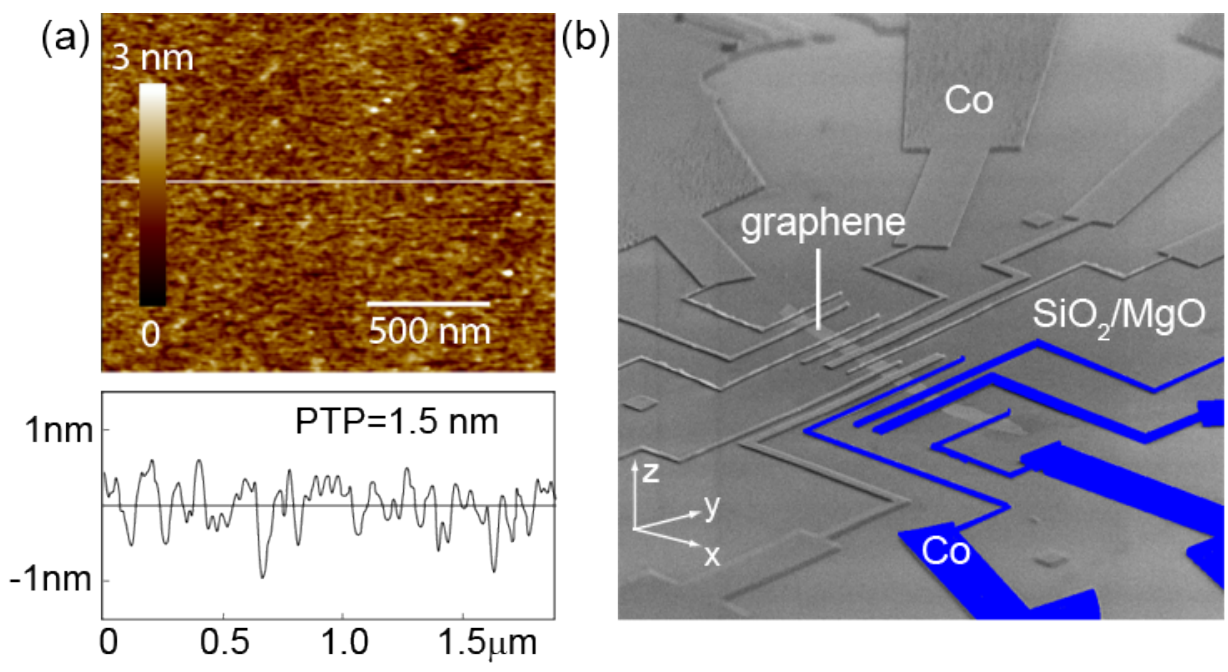

FIG. 1: (Color online) (a) AFM image of a BLG sample after MgO deposition: rms roughness $0.3 \mathrm{~nm}$. (b) SEM image of a BLG sample with multiple non-local spin valves.

The demonstration of micrometer long spin relaxation lengths in graphene by Tombros et al. 1] has made this two-dimensional material an extremely promising candidate for spintronics applications. So far most spin transport studies have focused on single layer graphene (SLG) [1-13] while the equally important bilayer graphene (BLG) has not yet received much attention. This is surprising since BLG has unique electronic properties which differ greatly from those of SLG (effective mass of carriers, electric-field induced band gap) and also differ from those of regular 2D electron gases (chirality) [14, 15]. It is currently believed that spin relaxation in SLG is limited by the momentum scattering from extrinsic impurities [2, 5, 16. Unlike SLG, the scattering from such charged impurities is reduced by the enhanced screening in BLG [17]. This leads to a relative importance of short-range (SR) scatterers in determining the transport properties such as the temperature $(T)$ and charge carrier density $(n)$ dependence of the BLG conductivity $(\sigma)$ [17 19]. In addition, interlayer hopping also plays an important role in the electronic properties of BLG and is predicted to cause an enhanced intrinsic spin-orbit ( $\mathrm{SO}$ ) coupling (up to $0.1 \mathrm{meV}$ in clean samples) in comparison to SLG [20]. Since charge and spin transport are highly linked, it is natural to expect a profound difference in the nature of spin transport in BLG. In particular, the unique electronic properties of BLG may offer new avenues to manipulate the spin degree of freedom.

In this Letter we report on spin transport studies in BLG both at room temperature 

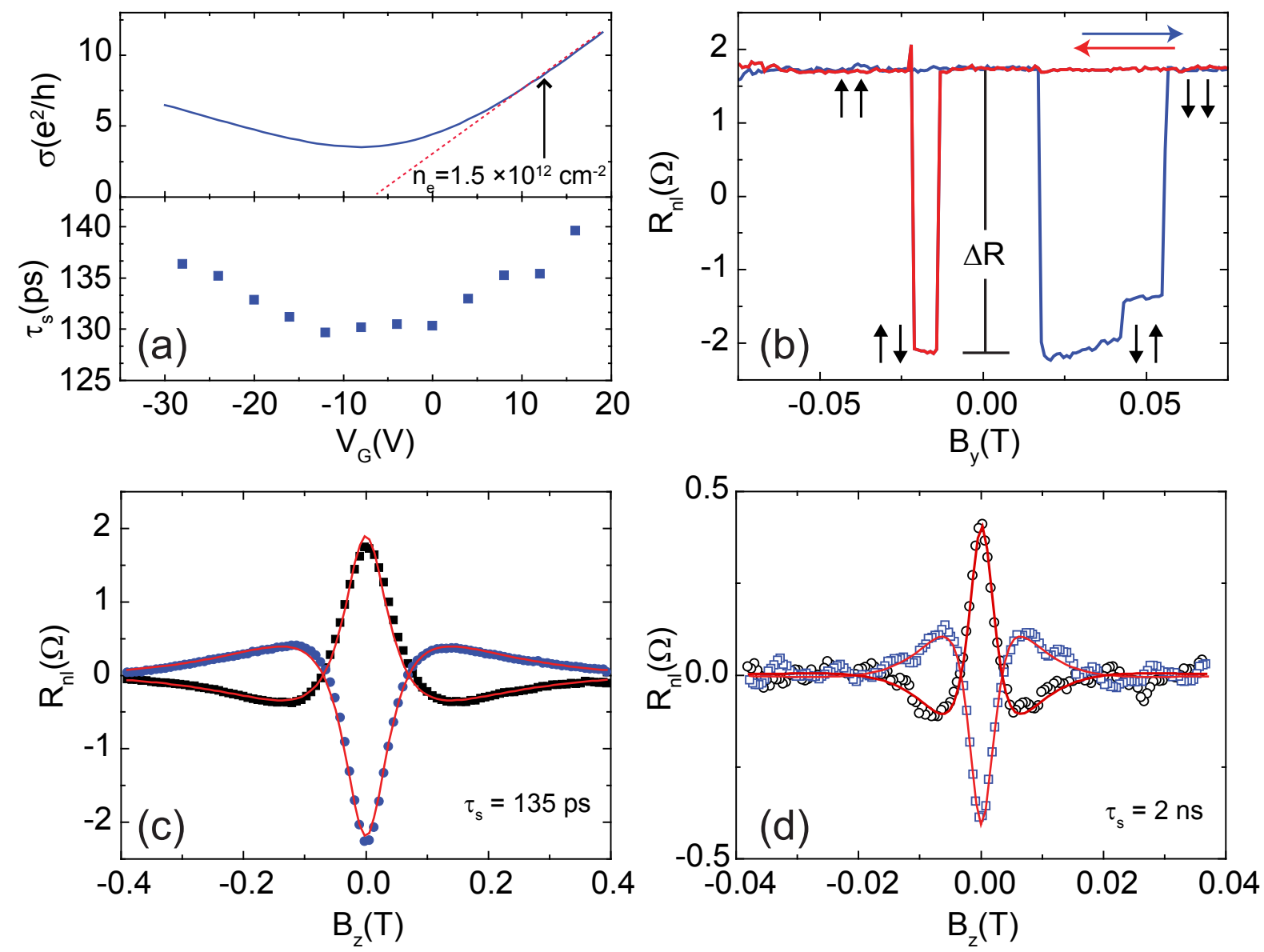

FIG. 2: (Color online) RT data: (a) $\sigma$ vs. $V_{G}$ and $\tau_{s}$ vs. $V_{G}$ for BLG. (b) Non-local resistance as a function of the in-plane magnetic field $B_{y}(T)$. The blue and red arrows show the field sweep direction while the black arrows show the relative magnetization orientations of the injector and detector electrodes. Hanle precession measurement for a perpendicular magnetic field $B_{z}(T)$ sweep for (c) the same sample with $\mu \sim 2000 \mathrm{~cm}^{2} / \mathrm{Vs}$ and (d) for a sample with $\mu \sim 300 \mathrm{~cm}^{2} / \mathrm{Vs}$.

(RT) and at low temperature (LT) using MgO barriers. Spin valve devices in the non-local geometry are fabricated on two types of $\mathrm{MgO}$-covered exfoliated graphene samples using standard e-beam lithography techniques. For global $\mathrm{MgO}$ samples, $\mathrm{MgO}$ covers the entire graphene surface while for local $\mathrm{MgO}$ samples the $\mathrm{MgO}$ is only under the Co electrodes. This is followed by the evaporation of the ferromagnetic contacts (Co) [21]. The AFM image after $\mathrm{MgO}$ deposition and the SEM image after the device fabrication for one of the BLG samples are shown in Fig.1. In order to investigate the nature of spin scattering in BLG, we have evaluated the spin relaxation time $\tau_{s}$ as a function of four paramters: (1) the 
field-effect mobility $\mu,(2)$ the minimum conductivity $\sigma_{\min }$, (3) the charge carrier density $n$ and (4) the temperature $T$ in the range of $5 \mathrm{~K}$ to $300 \mathrm{~K}$. Among these parameters the mobility dependence of $\tau_{s}$ provides the most direct way to deduce the dominant scattering mechanism: a linear dependence of $\tau_{s}$ on $\mu$ (or $\tau_{p}$ ) is a priori suggestive of an Elliott-Yafet (EY) spin scattering mechanism [22], while the inverse relation $\left(\tau_{s} \propto 1 / \mu \propto 1 / \tau_{p}\right)$ will indicate the dominance of D'yakonov-Perel' (DP) like spin scattering mechanisms [23]. In general, both mechanisms could be simultaneously relevant. For this study, we have selected representative 17 devices on 6 BLG samples whose field effect mobilities vary by more than one order of magnitude, from $\mu \sim 200 \mathrm{~cm}^{2} / \mathrm{Vs}$ to $8000 \mathrm{~cm}^{2} / \mathrm{Vs}$. The effect of impurity scattering on $\tau_{s}$ can also be deduced from $\sigma_{m i n}$, which depends on impurity concentration. The third parameter chosen is the charge carrier density: the density dependence of $\tau_{s}$ and $\tau_{p}$ is used to identify the spin scattering mechanism in SLG (EY). In BLG, the $\tau_{p}$ is often taken to be a constant under the assumption of charge scattering from weak short-range scatterers and charged impurities. Recent LT experiments [24, 25] indicate, however, that the density dependent $\tau_{p}$ results from strong short-range scattering in BLG. As a general trend in most of our samples $\sigma$ is linear at high carrier density $1-3 \times 10^{12} / \mathrm{cm}^{2}$ (see Fig. 2 upper panel). As the variation of $\tau_{p}$ is weak in this charge density range, we particularly compare the product $\tau_{s} \tau_{p}$ and the ratio $\tau_{s} / \tau_{p}$ as a function of carrier density $n$; the former is expected to be a constant for DP whereas the latter is a constant for EY. The fourth parameter chosen is temperature: unlike SLG, there is a strong temperature dependence of charge transport in BLG, which should also reflect itself in spin transport parameters.

Prior to any spin transport measurements, we have characterized the BLG device conductivity as a function of back gate voltage (Fig. 2a) using the standard four terminal geometry. As also observed by other groups [8, 10, the graphene samples are electron doped in the spin-valve configuration due to doping by the $\mathrm{Co} / \mathrm{MgO}$ barrier [26]. The non-local spin signal $(\triangle \mathrm{R})$ is measured by sweeping the in-plane magnetic field in a loop from negative $(-80 \mathrm{mT})$ to positive $(80 \mathrm{mT})$ and then back to negative values $(-80 \mathrm{mT})$ 27]. A clear bipolar spin transport signal is observed at RT (see Fig. 2b), with a positive value of the non-local resistance for parallel alignment of the electrodes' magnetization and a negative resistance for the anti-parallel alignment $(\Delta \mathrm{R}=4 \Omega)$. To confirm the observed spin signals, conventional Hanle spin precession measurements [28] are performed at the same electron density of $n=1.5 \times 10^{12} / \mathrm{cm}^{2}$ above which the conductance is linear and the mobility is 

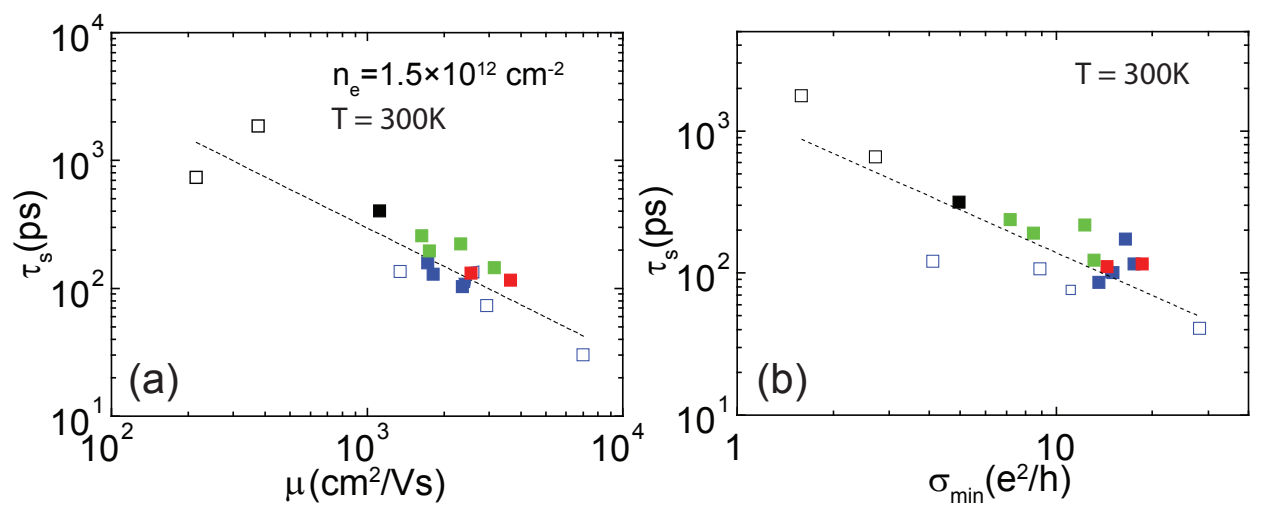

FIG. 3: (Color online) Results of Hanle precession measurements at RT for 17 BLG spin devices with mobility varying from $200-8000 \mathrm{~cm}^{2} /$ Vs. The data points with the same symbol represents different junctions on the same sample. Open and closed symbols correspond to samples with global and local $\mathrm{MgO}$, respectively. (a) $\tau_{s}$ taken at $n=1.5 \times 10^{12} / \mathrm{cm}^{2}$ vs. $\mu$ plotted on a $\log -\log$ scale. (b) $\tau_{s}$ taken at the CNP vs. $\sigma_{\min }$ for BLG samples at RT.

well defined within the Boltzmann approximation (Figs. 2c and 2d). The magnetic field dependence of the non-local resistance is fitted by

$$
R_{n l} \propto \int_{0}^{\infty} \frac{1}{\sqrt{4 \pi D_{s} t}} e^{\frac{-L^{2}}{4 D_{s} t}} \cos \left(\omega_{L} t\right) e^{\frac{-t}{\tau_{s}}} d t
$$

where $D_{s}$ is the spin diffusion coefficient and $\omega_{L}=\mathrm{g} \mu_{B} B / \hbar$ is the Larmor frequency for spin in an external magnetic field and thus gives the values for the spin parameters [28]. At $n=$ $1.5 \times 10^{12} / \mathrm{cm}^{2}$ we obtain a diffusion constant of $D_{s}=0.0032 \mathrm{~m}^{2} / \mathrm{s}$ and a spin relaxation time of $\tau_{s}=135 \mathrm{ps}$ for a sample with $\mu=\Delta \sigma / \mathrm{e} \Delta n=2000 \mathrm{~cm}^{2} / \mathrm{Vs}$ (Fig. 2c). The values give a spin relaxation length of $0.7 \mu \mathrm{m}$. The spin relaxation time $\tau_{s}$ as a function of gate voltage (doping) is plotted in Fig. 2a (lower panel) showing an increase $(<10 \%)$ of $\tau_{s}$ with doping, away from the charge neutrality point (CNP). This is qualitatively similar to the gate tunability of $\tau_{s}$ in SLG, although $\tau_{s}$ shows a weaker dependence in BLG at RT.

We next evaluate in detail the dependence of $\tau_{s}$ on $\mu$ in the Boltzmann regime. Such mobility dependent studies have been useful in identifying spin scattering mechanisms in inorganic semiconductor systems [29]. As shown in Fig. 3a by a log-log plot, we observe an inverse dependence of $\tau_{s}$ on the mobility. Note that all data are taken at an electron density $n=1.5 \times 10^{12} / \mathrm{cm}^{2}$. In samples with the highest mobility $\tau_{s}$ is only $30 \mathrm{ps}$. On the other hand, we observe a spin relaxation time $\tau_{s}$ of up to 2 ns at RT for samples with the lowest mobilities (see corresponding Hanle curve in Fig. 2d, [30]). Such values 
for $\tau_{s}$ are one order of magnitude longer compared to values reported so far in any SLG experiment. Furthermore, this strong variation of $\tau_{s}$ with $\mu$ offers the most direct evidence of the correlation between spin and charge transport. Since higher mobilty samples will typically involve higher momentum relaxation time $\tau_{p}$, assuming $\mu \propto \tau_{p}$ in the Boltzmann regime, the inverse dependence of $\tau_{s}$ on $\mu$ clearly demonstrates that the DP mechanism is the dominant spin scattering mechanism in BLG at RT.

The same plot of $\tau_{s}$ vs. $\mu$ is also useful to elucidate the possible origin of the DP mechanism in our samples. For this, we first estimate the strength of spin-orbit (SO) coupling $(\Delta)$ in BLG using the expression: $1 / \tau_{s}=\Omega_{e f f}^{2} \tau_{p}=4 \Delta^{2} \tau_{p} / \hbar^{2}$, where $\Omega_{e f f}$ is the effective Larmor frequency of the precessing spins and $\Delta$ is the corresponding SO coupling strength [16]. The value $\Omega_{\text {eff }}$ is obtained from the $\tau_{s}$ vs. $\mu$ data (Fig. 3a), $\Omega_{\text {eff }}=407 \pm 25 \mathrm{GHz}$, which gives $\Delta \sim 0.14 \pm 0.01 \mathrm{meV}$. Additionally, $\Delta$ only weakly depends on temperature [21]. Therefore, it is unlikely that low energy phonons (such as acoustic phonons) are responsible for the observed spin scattering. The most important open question is whether this SO coupling is intrinsic or extrinsic in nature. The intrinsic SO coupling of BLG is expected to lead to $\Delta_{\text {intrinsic }}$ up to $0.1 \mathrm{meV}$ in clean BLG samples [20]. While this is in good agreement with the value extracted from the $\tau_{s}$ vs. $\mu$ plot, the influence of both the externally applied electric field and the role of adatoms cannot be excluded. Since interlayer hopping is involved in BLG, electric field dependent modifications to the intrinsic SO coupling are expected. Adatoms, on the other hand, induce local curvature to an otherwise flat graphene lattice and can cause spin scattering by both EY and DP mechanism [12, 16]. In SLG, the recent studies on the influence of external adatoms on spin transport also shows an increase in $\tau_{s}$ with increase in adatom concentration indicating a DP like scattering at LT [9]. However, in the case of BLG the role of the adatoms, in determining DP or EY spin scattering, might be even smaller due to a higher lattice stiffness [31], thus reducing the adatom induced SO coupling strength. Moreover, the effect of the charged impurities is also reduced due to enhanced screening in BLG. Thus the two prominent factors (charged impurities and adatoms) responsible for the spin scattering in SLG play a minor role in BLG which could also be the reason why we see longer spin relaxation times in BLG. More investigations are needed to clarify the type and concentration of spin scatterers in single and bilayer graphene and to differentiate the contribution from extrinsic and intrinsic factors to the SO coupling in BLG samples with disorder. 

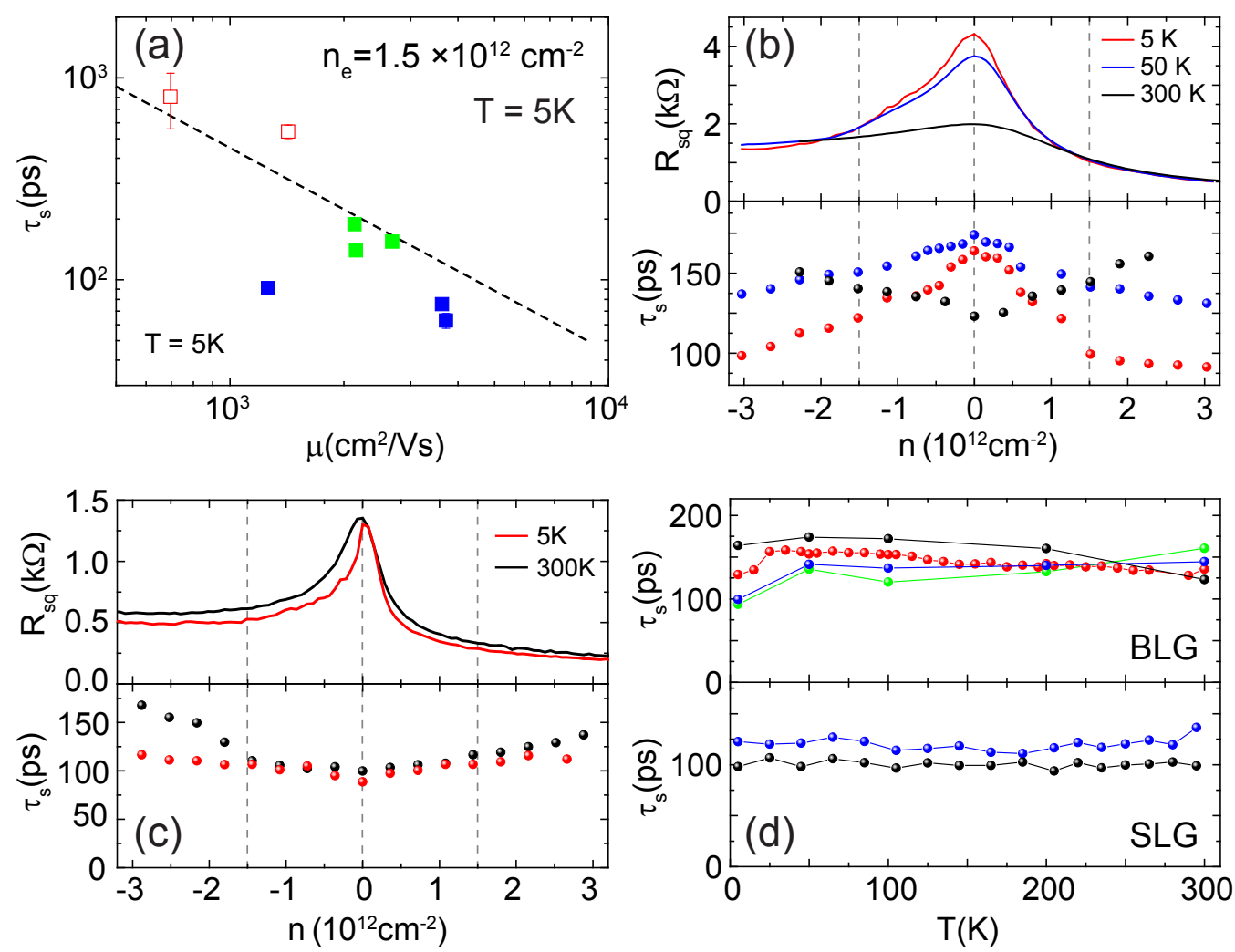

FIG. 4: (Color online) (a) $\tau_{s}$ vs. $\mu$ for 8 BLG junctions at $5 \mathrm{~K}$. (b) Upper panel: $R$ vs. $n$ for BLG; Lower panel: $\tau_{s}$ vs. $n$ for $T=300 \mathrm{~K}$ (black circles), $50 \mathrm{~K}$ (blue circles) and $5 \mathrm{~K}$ (red circles). (c) Upper panel: $R$ vs. $n$ for SLG; Lower panel: $\tau_{s}$ vs. $n$ for SLG at RT (black circles) and at $5 \mathrm{~K}$ (red circles). (d) Upper panel: $\tau_{s}$ vs. $T$ for four densities, $n=\mathrm{CNP}$ (black circles), 0.7 (red circles), 1.5 (blue circles) and 2.2 (green circles) $\times 10^{12} / \mathrm{cm}^{2}$. Lower panel: $\tau_{s}$ vs. $T$ for SLG at $n=\mathrm{CNP}$ (black circles), 1.5 (blue circles) $\times 10^{12} / \mathrm{cm}^{2}$.

We next evaluate the dependence of $\tau_{s}$ on the RT minimum conductivity $\sigma_{\text {min }}$ and charge carrier density $n$ of the BLG samples. The $\tau_{s}$ vs. $\sigma_{\text {min }}$ graph (Fig. 3b) indicates a decrease in $\tau_{s}$ with increasing $\sigma_{m i n}$. The value of $\sigma_{\min }$ at RT varies significantly between clean and dirty samples and is higher for cleaner samples [19, 32, 33]. The inverse dependence of $\tau_{s}$ on $\sigma_{\min }$ indicates a higher spin relaxation time in dirtier samples. Therefore, this correlation is in good agreement with the above conclusion that DP spin scattering is dominant in BLG at RT. Finally, we analyze the weak dependence of $\tau_{s}$ on $n$ for individual samples (Fig. 2a). At first we note that at RT the density dependence of $\tau_{p}=\sigma m^{*} / n \mathrm{e}^{2}$ shows a gradual decrease with increasing $n$ in the density range $1-3 \times 10^{12} / \mathrm{cm}^{2}$ for our samples [21]. In this range, 
the quantity $\tau_{s} \tau_{p}$ is almost constant ( $\sim 4 \%$ change with charge density) while $\tau_{s} / \tau_{p}$ shows an increase of $28 \%$ with increasing charge density at RT. This is consistent with the dominance of the DP mechanism at RT.

So far we have evaluated spin transport at RT. Next we evaluate spin transport as a function of temperature. Here, it is important to note that for BLG (Fig. 4b), and unlike SLG (Fig. 4c), the charge transport already shows strong changes with decreasing temperature due to the thermally activated nature of carriers near the CNP [21, 34, 35]. We may expect these changes to be reflected in the spin transport, as the temperature is lowered. We first note that for charge transport, there are two distinct bilayer specific density regimes (Fig. 4(b) upper panel): (A) $n>1 \times 10^{12} / \mathrm{cm}^{2}$, involving temperature independent transport and (B) $n<1 \times 10^{12} / \mathrm{cm}^{2}$, involving thermally-activated transport [21]. For regime (A) away from the CNP the mobility is well defined. Here the $\tau_{s}$ can be easily evaluated, similar to the approach used for analyzing RT data. We measured 8 devices in 3 samples with $\mu$ ranging from $700 \mathrm{~cm}^{2} / \mathrm{Vs}$ to $3800 \mathrm{~cm}^{2} / \mathrm{Vs}$ at $T=5 \mathrm{~K}$. The data plotted in Fig. 4a, show that the inverse dependence of $\tau_{s}$ on $\mu$ persists down to $5 \mathrm{~K}$, demonstrating $\mathrm{DP}$ as the dominant spin scattering mechanism even at LT. At the same time, we note that the density dependence of $\tau_{s}$ and $\tau_{p}$ is rather weak in the Boltzmann regime for $n>1 \times 10^{12} / \mathrm{cm}^{2}$ and does not allow for a clear assignment of the dominant spin scattering mechanism. The quantities $\tau_{s} \tau_{p}$ and $\tau_{s} / \tau_{p}$ show comparable small changes in this regime both at $50 \mathrm{~K}$ and at $5 \mathrm{~K}$ [21]. Therefore, in this density regime we are left only with the mobility dependence at LT.

We finally consider the low density regime $\left(n<1 \times 10^{12} / \mathrm{cm}^{2}\right)$ around the CNP, involving the thermally activated behavior of the charge transport. Here, the minimum conductivity at $\operatorname{CNP}\left(\sigma_{\min }\right)$ is not any more a suitable parameter for scaling $\tau_{s}$. The available $\sigma_{\text {min }}$ data scatters significantly at LT (not shown), but the overall variation in $\sigma_{\min }$ is small compared to RT. This is not surprising since $\sigma_{\min }$ is expected to take a disorder independent value of $3 \mathrm{e}^{2} / \pi \mathrm{h}$ at $T=0 \mathrm{~K}\left[36\right.$. Thus we are left only with the density dependence of $\tau_{s}$ to elucidate on what happens near the CNP at LT. This density dependence of $\tau_{s}$ at RT shows a minimum at CNP, as discussed above. As the temperature is lowered, this minimum in $\tau_{s}$ is gradually suppressed and the slope of $\tau_{s}(n)$ changes sign. Finally, at $T=5 \mathrm{~K}$ the density dependence of $\tau_{s}$ shows strong enhancement (> $50 \%$ ) near the CNP (Fig. 4b, lower panel).

The key to understanding which spin scattering mechanism dominates at LT near CNP 
lies in the density dependence of $\tau_{p}$. We note that $\tau_{p}=\sigma \mathrm{m}^{*} / n \mathrm{e}^{2}$ (Boltzmann approximation), estimated for our samples, gives a quantitative estimate only in the high density (Boltzmann) regime. Closer to the CNP, $\tau_{p}$ extracted from the above assumption shows an increase with decreasing density [21]. Recent detailed experiments on BLG at LT have shown that a divergence of $\tau_{p}$ near the CNP is indeed observed [21, 24, 25]. With this, a correlation of $\tau_{s}(n)$ with $\tau_{p}(n)$ suggests a transition from DP to EY spin scattering mechanism at LT, around the CNP [21]. Since the momentum scattering mechanism is different near CNP at LT, it is not surprising that the spin scattering mechanism is also different. We note that two mysteries remain to be resolved: (1) why is a transition to the different spin scattering mechanism observed in BLG (1) near CNP, and (2) only at LT. One possible explanation could be related to the thermally activated nature of carriers in this density regime.

In conclusion, we have demonstrated spin injection and detection in BLG across MgO barriers and observed spin relaxation times up to $2 \mathrm{~ns}$ at room temperature. Our systematic study shows that at RT spin scattering in BLG follows an inverse dependence of $\tau_{s}$ on both the mobilty $\mu$ and the room temperature $\sigma_{m i n}$, indicating a DP spin scattering mechanism. We disuss the role of intrinsic and extrinsic factors that could lead to the dominance of DP spin scattering in BLG. While the inverse scaling of mobility with $\tau_{s}$ persists down to $5 \mathrm{~K}$, the density dependence of $\tau_{s}$ indicates deviations from DP mechanism at these low temperatures near the charge neutrality point.

The Aachen team acknowledges support by DFG through FOR 912. B.B and M.P. acknowledge support from JARA-FIT. The Singapore team acknowledges support by the Singapore National Research Foundation under NRF RF Award No. NRF-RF2008-07, NRFR-143-000-360-281, US Office of Naval Research (ONR and ONR Global), and by NUS NanoCore.

Note added: After the submission of this manuscript, related works on BLG and on multilayer graphene became available [37, 38].

[1] N. Tombros et al., Nature 448, 571 (2007).

[2] N. Tombros et al., Phys. Rev. Lett. 101, 046601 (2008).

[3] C. Jozsa et al., Phys. Rev. Lett. 100, 236603 (2008). 
[4] M. Popinciuc et al., Phys. Rev. B 80, 214427 (2009).

[5] C. Jozsa et al., Phys. Rev. B 80, 241403 (2009).

[6] S. Cho, Y-F. Chen, and M.S. Fuhrer, Appl. Phys. Lett. 91, 123105 (2007)

[7] W. Han et al., Appl. Phys. Lett. 94, 222109 (2009).

[8] W. Han et al., Phys. Rev. Lett. 102, 137205 (2009).

[9] K. Pi et al., Phys. Rev. Lett. 104, 187201 (2010).

[10] W. Han et al., Phys. Rev. Lett. 105, 167202 (2010).

[11] M. Shiraishi et al., Adv. Funct. Mater. 19, 3711 (2009).

[12] A.H. Castro Neto and F. Guinea, Phys. Rev. Lett. 103, 026804 (2009).

[13] D. Huertas-Hernando et al., Phys. Rev. Lett. 103, 146801 (2009).

[14] K.S. Novoselov et al., Science 306, 666 (2004).

[15] A.H.C. Neto et al., Rev. Mod. Phys. 81, 109 (2009).

[16] C. Ertler et al., Phys. Rev. B 80, 041405 (2009).

[17] S. Das Sarma et al., Phys. Rev. B 81, 161407 (2010).

[18] S. Das Sarma et al., arXiv:1003.4731v1.

[19] S. Adam and M. D. Stiles, arXiv:0912.1606v2 (2010).

[20] F. Guinea, New Journal of Physics 12, 083063 (2010).

[21] See supplementary material for detailed descriptions.

[22] R.J. Elliott, Phys. Review 96, 266(1954).

[23] M.I. D'yakonov and V.I. Perel, Sov. Phys. Solid State 13, 3023 (1972).

[24] Y.-W. Tan et al., Phys. Rev. Lett. 99, 246803 (2007).

[25] M. Monteverde et al., Phys. Rev. Lett. 104, 126801 (2010).

[26] In addition the Co electrodes induce some distortion on the hole side of the $\mathrm{R}_{s q} \mathrm{vs} . \mathrm{V}_{G}$.

[27] F.J. Jedema et al., Nature 416, 713 (2002).

[28] F.J. Jedema et al., Appl. Phys. Lett. 81, 5162 (2002).

[29] R.I. Dzhioev et al., Phys. Rev. Lett. 93, 216402 (2004).

[30] We note that there are small amplitude oscillations on top of the Hanle signal, which we only observe for the sample with the longest $\tau_{s}$. A similar effect has been reported in Ref. [2].

[31] J.C. Meyer et al., Solid State Commun. 143, 101 (2007).

[32] T. Stauber, N.M.R. Peres and F. Guinea, Phys. Rev. B 76, 205423 (2007).

[33] S. Xiao et al., Phys. Rev. B 82, 041406 (2010). 
[34] S.V. Morozov et al., Phys. Rev. Lett. 100, 016602 (2008).

[35] S. Adam and S.Das Sarma, Phys. Rev. B 77, 115436 (2008).

[36] J. Nilsson et al., Phys. Rev. Lett. 97, 266801 (2006).

[37] W. Han and R. K. Kawakami, arXiv: 1012.3435 (2010)

[38] T. Maassen et al., Phys. Rev. B 83, 115410 (2011). 
Supplementary Information

\section{Observation of Long Spin Relaxation Times in Bilayer Graphene at Room}

\section{Temperature}

T. - Y. Yang, ${ }^{1,2, *}$ J. Balakrishnan, ${ }^{3, *}$ F. Volmer, ${ }^{1,2}$ A. Avsar, ${ }^{3}$ M. Jaiswal, ${ }^{3}$ J. Samm, ${ }^{1,2}$

S. R. Ali, ${ }^{1,2}$ A.Pachoud, ${ }^{3}$ M. Zeng, ${ }^{3}$ M. Popinciuc, ${ }^{1,2}$ G. Güntherodt, ${ }^{1,2}$ B. Beschoten, ${ }^{1,2,}$ and B. Özyilmaz ${ }^{3,4 \dagger}$

${ }^{1}$ II. Institute of Physics, RWTH Aachen University, 52074 Aachen, Germany

${ }^{2}$ JARA: Fundamentals of Future Information Technology, 52074 Aachen, Germany

${ }^{3}$ Department of Physics, National University of Singapore, Singapore 117542, Singapore

${ }^{4}$ NanoCore, National University of Singapore, Singapore 117576, Singapore

* These authors contributed equally to this work

†e-mail: barbaros@nus.edu.sg; bernd.beschoten@physik.rwth-aachen.de

\section{BLG spin valve device Fabrication:}

The graphene samples for our devices are prepared by micromechanical exfoliation of graphite onto a $\mathrm{Si} / \mathrm{SiO}_{2}$ substrate. Both $\mathrm{SLG}$ and BLG samples are first identified by their optical contrast which is then confirmed by Raman spectroscopy (laser wavelength $=514 \mathrm{~nm}$ ) (see Fig $\mathrm{S} 1 b)$. For global $\mathrm{MgO}$ samples ( $\mathrm{MgO}$ covered over the entire graphene surface), the next step is to deposit $\mathrm{MgO}$ under molecular beam epitaxy (MBE) conditions followed by standard e-beam lithography for patterning the sample. Co electrodes are then deposited by MBE. For local MgO samples ( $\mathrm{MgO}$ under the Co electrodes only), we use first e-beam lithography for electrode patterning. This is followed by a single run deposition of $\mathrm{MgO} / \mathrm{Co}$ under identical $\mathrm{MBE}$ 
conditions. A thin film of $\mathrm{MgO}(1-2 \mathrm{~nm})$ is deposited between Co and graphene to overcome the conductivity mismatch and to increase the injection efficiency of spin polarized carriers. Prior to and after $\mathrm{MgO}$ deposition (deposition rate of $0.007 \mathrm{~nm} / \mathrm{s}$ ), the samples were annealed for one hour at $200^{\circ} \mathrm{C}$ in vacuum $\left(5 \times 10^{-10} \mathrm{mbar}\right)$. The pre- and post-annealing steps allow a uniform $\mathrm{MgO}$ growth with typical thickness of $2 \mathrm{~nm}$ and roughness of around $\sim 0.3 \mathrm{~nm}$ on the graphene surface (see Fig. 1a). Although we do not use $\mathrm{TiO}$ [S1] as a buffer layer for uniform $\mathrm{MgO}$ growth, we do obtain a uniform $\mathrm{MgO}$ layer but with pin holes in some of our $\mathrm{MgO}$ barriers. Standard e-beam lithography is performed to write the electrode patterns. The widths of the FM electrodes in our devices are between $300 \mathrm{~nm}$ to $1 \mu \mathrm{m}$ with the separation between the electrodes in the range $0.50 \mu \mathrm{m}$ to $2.6 \mu \mathrm{m}$. In a single run we deposit a $30-40 \mathrm{~nm}$ thick Co layer for the electrodes and contact pads.

It is also important to note that after $\mathrm{MgO}$ deposition, we do not see any amorphization of graphene as reported by other groups [S2]. In Ref. [S2], the authors use sputter deposition for $\mathrm{Al}_{2} \mathrm{O}_{3}$ and $\mathrm{MgO}$ layers. In contrast, we have performed $\mathrm{MBE}$ growth of $\mathrm{MgO}$. Although we cannot directly compare both techniques, we expect that the graphene layer structure is less affected during the MBE growth of MgO. This is confirmed by Raman spectroscopy. Figure S1b shows one such Raman spectrum with the characteristic $\mathrm{G}$ and 2D peaks of graphene after $\mathrm{MgO}$ deposition confirming the quality of graphene samples even after $\mathrm{MgO}$ deposition. 

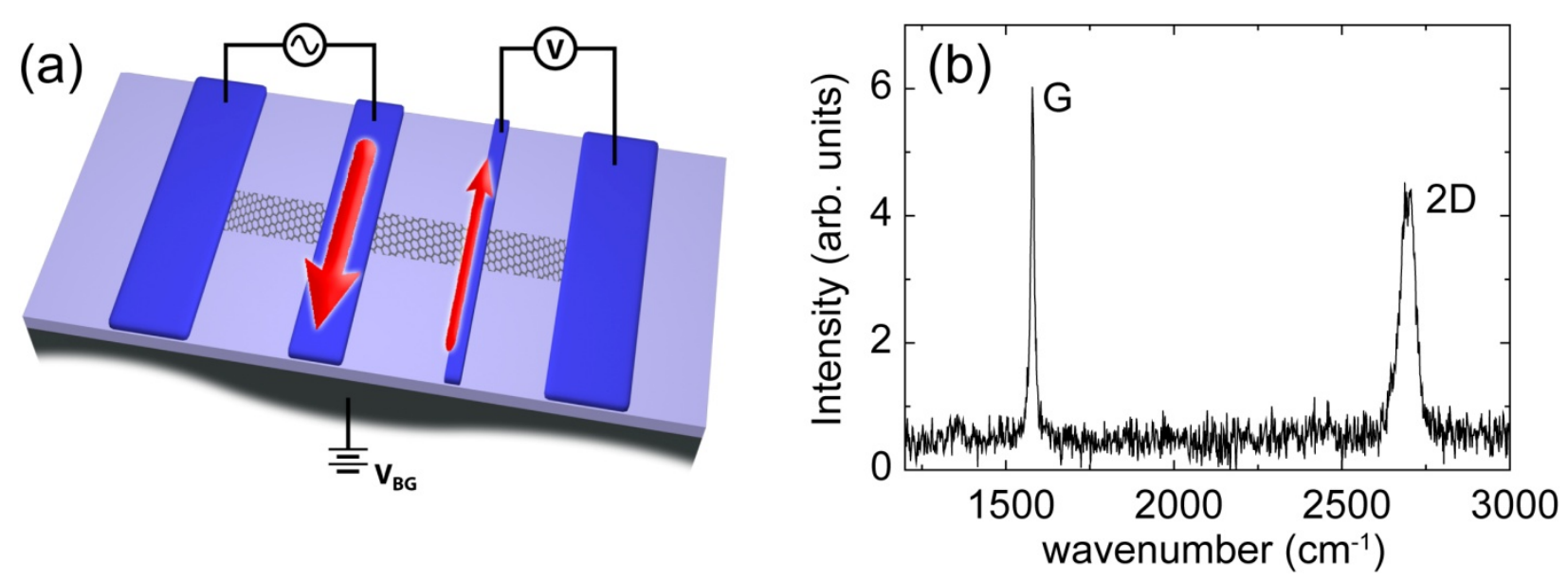

Figure S1: (a) Schematic of a graphene based non-local spin valve. Measurements are performed

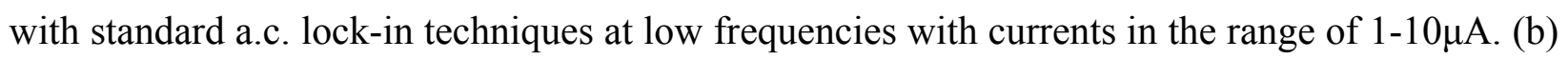
Typical Raman spectrum of a MgO covered bilayer sample with $\mathrm{G}$ peak at $1579 \mathrm{~cm}^{-1}$ and 2D peak at $2695 \mathrm{~cm}^{-1}$ with FWHM $\sim 55$.

\section{Temperature Dependence of BLG resistivity.}

In BLG, the conductivity minimum at the charge neutrality point (CNP) shows a distinct behaviour when compared to SLG (see fig. S2). The reason for such an increase in the device resistivity with lowering temperature is attributed to the thermally activated nature of charge carriers near CNP [S3, S4]. This can be confirmed by analyzing the temperature dependence of conductivity $\sigma$ at different densities. Figure S2 (b) shows the temperature dependence of $\sigma$ at two different densities $\mathrm{n}\left(\mathrm{CNP}, 1 \times 10^{12} / \mathrm{cm}^{2}\right)$, indicating an insulating behaviour at low $\mathrm{T}$ [S2]. 

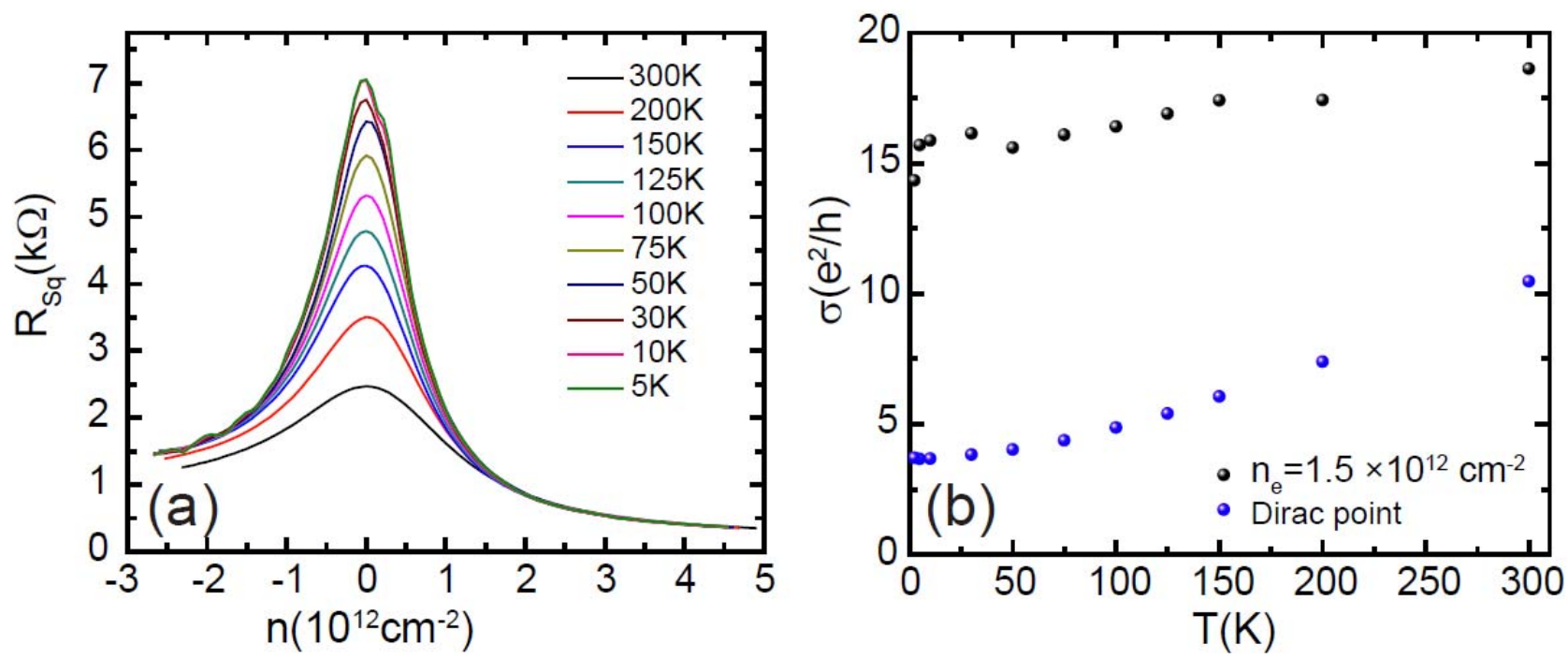

Figure S2: (a) Carrier density dependence of BLG resistivity for the temperature range $5-300$

K. (b) Temperature dependence of conductivity at the CNP and at $n=1.5 \times 10^{12} / \mathrm{cm}^{2}$.

\section{Temperature Dependence of $\tau_{\underline{s}}$ and $\tau_{\mathrm{p}}$ for BLG:}

Determining the relation between the spin relaxation time and momentum relaxation time is essential to deduce the dominant spin scattering mechanism: a linear relation, $\tau_{\mathrm{s}} \alpha \tau_{\mathrm{p}}$ indicates the dominance of Elliott-Yafet (EY) spin scattering, while the reciprocal relation suggests the dominance D'yakonov-Perel' (DP) like spin scattering. Hence we plot the product $\tau_{\mathrm{s}} \tau_{\mathrm{p}}$ and the ratio $\tau_{\mathrm{s}} / \tau_{\mathrm{p}}$ as a function of density at different temperatures [Fig S3 (b, c, \& d)]. Fig. S3 (a) shows the variation of $\tau_{\mathrm{p}}$ as a function of carrier density. The estimate of $\tau_{\mathrm{p}}$ assumes Boltzmann transport, $\tau_{\mathrm{p}}=\sigma \mathrm{m}^{*} / \mathrm{ne}^{2}$ (where $\mathrm{m}^{*}=0.03 \mathrm{~m}_{\mathrm{e}}$ for BLG). The strong increase of $\tau_{\mathrm{p}}$ near the CNP is qualitatively in good agreement with the results obtained from recent experiments on BLG [S5, S6]. This increase has been attributed to logarithmic corrections to $\tau_{\mathrm{p}}$ arising from the presence of strong short-range scatterers. Therefore, it is reasonable to assume a similar scaling of $\tau_{\mathrm{p}}(n)$ in our samples at LT near the CNP. The values for $\tau_{\mathrm{s}} \tau_{\mathrm{p}}$ at $300 \mathrm{~K}$ as function of carrier density in 
Fig. S3b are almost constant ( $4 \%$ change with carrier density). This confirms the observed DP scattering. On the other hand, the ratio $\tau_{\mathrm{s}} / \tau_{\mathrm{p}}$ shows a significant increase of $28 \%$ at room temperature. However, at LT both the quantities vary by a comparable amount, i.e. both $\tau_{\mathrm{s}} \tau_{\mathrm{p}}$ and $\tau_{\mathrm{s}} / \tau_{\mathrm{p}}$ show in the density range $1-3 \times 10^{12} / \mathrm{cm}^{2}$ a decrease by $\sim 10 \%$ at $50 \mathrm{~K}$ and by $\sim 25 \%$ at 5 $\mathrm{K}$. This makes it difficult to deduce the dominant scattering mechanism from density dependence alone. Therefore, at LT the scattering mechanism is determined only by the mobility dependence of the spin relaxation time. Moreover, it should be noted that the dependencies of $\tau_{\mathrm{s}} \tau_{\mathrm{p}}$ and $\tau_{\mathrm{s}} / \tau_{\mathrm{p}}$ given here are consistently observed in all our samples.

Effect of electron-hole puddles at CNP in BLG at LT: As discussed, the logarithmic corrections arising from the presence of strong short-range scatterers, causes a strong increase in $\tau_{\mathrm{p}}$ near the CNP in BLG. Thus, a comparison of the $\tau_{\mathrm{p}}$ and $\tau_{\mathrm{s}}$ values indicates a transition from DP to EY like spin scattering in BLG at LT near the CNP. Moreover, the presence of electron-hole puddles for these bias voltages may also suggest the possibility of spin scattering via the Bir-AronovPikus (BAP) mechanism [S7]. If such electron-hole scattering together with spin flip is present, the spin scattering is expected to happen only at the boundaries of electron-hole puddles and not uniformly across the samples. This is because the BAP spin scattering requires a significant overlap in the electron and hole wave functions. Although we cannot completely rule out spin scattering of BAP-type, we believe that this is not likely because it is usually only relevant in hole-doped systems with large effective mass of the charge carriers [S8]. However, precise theoretical studies on the electron-hole scattering near the CNP are required to fully estimate the influence of electron-hole exchange interaction in spin scattering. 

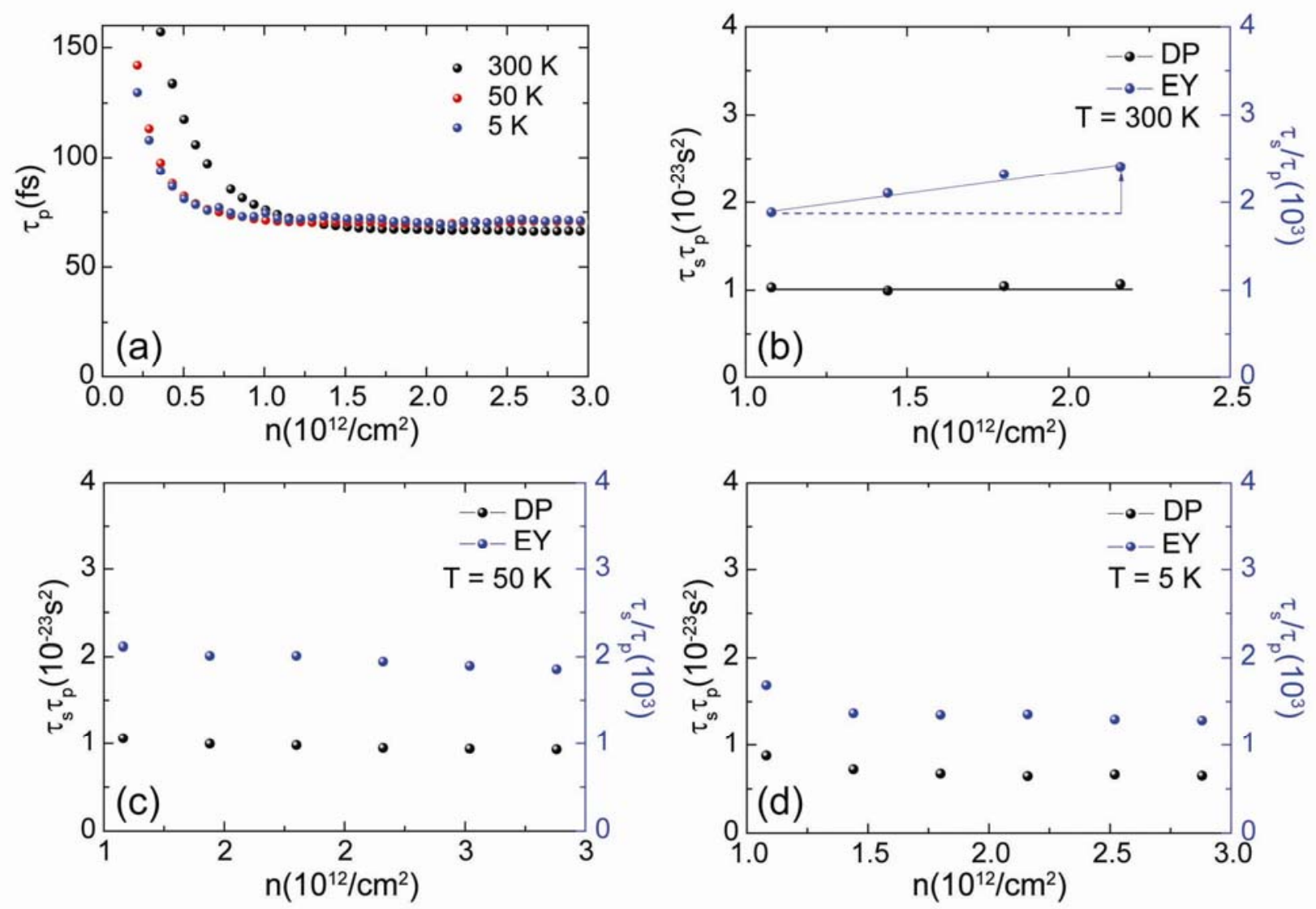

Figure S3: (a) The momentum relaxation time, $\tau_{p}=\sigma m * / n e^{2}$ calculated in the Boltzmann framework as a function of carrier density $n$ for BLG at $T=300 \mathrm{~K}$ (black circles), $50 \mathrm{~K}$ (red circles) and $5 \mathrm{~K}$ (blue circles); (b, c and d) The carrier density dependence of the product $\tau_{\mathrm{s}} \tau_{\mathrm{p}}$ and the ratio $\tau_{\mathrm{s}} / \tau_{\mathrm{p}}$, which identifies the dominant scattering mechanism for $T=300 \mathrm{~K}, 50 \mathrm{~K}$ and $5 \mathrm{~K}$, respectively. A constant value for $\tau_{\mathrm{s}} \tau_{\mathrm{p}}$ indicates DP while a constant value for $\tau_{\mathrm{s}} / \tau_{\mathrm{p}}$ indicates EY mechanism. The arrow in fig $\mathrm{b}$ shows the significant change in the ratio $\tau_{\mathrm{s}} / \tau_{\mathrm{p}}$ with density at $\mathrm{RT}$ when compared to the change in $\tau_{\mathrm{s}} \tau_{\mathrm{p}}$

IV. Determination of the strength of spin-orbit coupling as a function of Mobility and temperature. 
The spin-orbit coupling $(\Delta)$ is estimated from the Larmor frequency as $\Delta=\Omega_{\text {eff }} \hbar / 2$ [S6]. Here the Larmor frequency is defined as $\Omega_{\text {eff }}^{2}=1 / \tau_{s} \tau_{p}$ and is evaluated from fig. $3 a \& 3 b$ (see main text). The values of $\Delta$ thus obtained show only weak temperature dependence $(0.14 \pm 0.01 \mathrm{meV}$ at RT and $0.12 \pm 0.009 \mathrm{meV}$ at LT) and mobility dependence. This eliminates the possible contribution from low energy phonons and suggests contributions from adatoms and from intrinsic spin-orbit coupling to the observed spin scattering in BLG. Furthermore, the SO coupling arising from phonons (acoustic and surface-optical phonons) and the charged impurities in $\mathrm{SiO}_{2}$ have been estimated from first principle calculations for SLG. Both these extrinsic factors were shown to cause SO coupling weaker by orders of magnitude [S9] than required for the observation of subnanosecond spin scattering times in SLG.

\section{$\underline{\text { References }}$}

[S1] W. Han et al., Phys. Rev. Lett. 105, 167202 (2010).

[S2] B. Dlubak et al. Appl. Phys. Lett. 97, 092502 (2010).

[S3] S. V. Morozov et al., Phys. Rev. Lett. 100, 016602 (2008).

[S4] S. Adam and S. Das Sarma, Phys. Rev. B 77, 115436 (2008).

[S5] Y.-W. Tan et al., Phys. Rev. Lett. 99, 246803 (2007).

[S6] M. Monteverde et al., Phys. Rev. Lett. 104, 126801 (2010).

[S7] G. L. Bir, A. G. Aronov, and G. E. Pikus, Zh. Eksp. Teor. Fiz. 69,1382 (1975), Sov. Phys. JETP 42, 705 (1975).

[S8] Igor Žutić, Jaroslav Fabian, and S. Das Sarma, Rev. Mod. Phys. 76, 323 (2004).

[S9] C. Ertler et al., Phys. Rev. B 80, 041405 (2009). 
